\title{
Ideal care and the realities of practice: interdisciplinary relationships in the management of advanced cancer patients in Australian emergency departments
}

\author{
Authors: Lane H, Weil J, Jelinek GA, Boughey M, Marck CH, Weiland TJ, Haydon A, Philip J \\ Corresponding author: Dr Heather Lane, Centre for Palliative Care, St Vincent's Hospital and the \\ University of Melbourne, P.O. Box 2900, Fitzroy 3065, Victoria, Australia
}

Email: heather.lane@svhm.org.au

Phone: +61394160000

\section{Background}

Over the course of their illness, a person with cancer is likely to see a number of different doctors including a general practitioner, surgeon, medical oncologist, radiation oncologist and possibly a palliative care physician. Co-ordination of cancer care has been recognised as important to the optimal delivery of cancer services. To aid coordination and improve collegial interaction in cancer services cancer multidisciplinary meetings, in which medical specialists and allied health staff working in cancer care meet to discuss and plan care, have become recommended internationally and recognised as standard of care $[1,2,3]$.

However, challenges in interdisciplinary communication persist. Patients perceive information flow from the hospital to the community to be poor [4]. General practitioners report that the communication received when their patients are discharged from hospitals is often delayed [5] and fails to include potentially important information [6,7]. Meanwhile, oncologists note that referral letters from general practitioners frequently lack information they consider important [7].

It is not just general practitioners and cancer specialists who provide care to people with cancer. Patients with advancing disease frequently access palliative care expertise. In Australia, palliative care may be provided at home (through community palliative care services), in an acute hospital (through hospital consultancy palliative care services) and in inpatient palliative care units (or hospice). Palliative care provision is not based on expected prognosis and can be provided alongside anti-cancer treatments, such as chemotherapy or radiotherapy. The effective transfer of information between health care providers is essential for seamless care delivery.

Emergency department (ED) healthcare professionals are also frequently involved in the care of cancer patients, often at times of crisis. An Australian study reported 469 unplanned hospital presentations were made by 316 outpatients receiving chemotherapy [8], while patients with primary 
malignant glioma have a median of three ED visits, spending a median of 13 hours in ED, between diagnosis and death [9].

Whilst the experience and views of ED staff caring for advanced cancer patients in the ED has received some attention [10, 11], there is limited research examining the interaction between the involved healthcare professionals when such a patient presents to the ED.

This qualitative study aimed to explore healthcare professionals' views and experiences of interdisciplinary interactions when caring for patients with advanced cancer who present to the ED. For the purpose of this study, interdisciplinary interaction was defined as interaction between people working in separate medical specialty areas, such as emergency medicine, oncology and palliative care.

\section{Method}

\section{Participants}

Participants included clinical staff working in ED, oncology, and hospital-based palliative care services from two quaternary referral hospitals and clinicians working in two community palliative care services in Melbourne, Australia. Clinical staff working in each service were invited to participate in focus groups, with invitations and focus group scheduling facilitated by a senior staff member in participating services.

To broaden the range of participants and ensure the broadest range of views were explored, emergency physicians and emergency nurses from other Australian states were also included using supplemental semi-structured telephone interviews. An email invitation was sent to all members of the Australasian College for Emergency Medicine and the College of Emergency Nursing Australia. The interested respondents were purposively sampled to include participants from a variety of different centre sizes (e.g. urban, regional, and rural) and to ensure each of the five Australian States and two Territories were sampled.

\section{Data Collection}

A series of focus groups were conducted with Melbourne based clinical staff, with separate focus groups conducted for each medical specialty area, for example, emergency medicine, oncology, hospital-based palliative care and community-based palliative care. Focus groups were conducted at the participants' place of work and facilitated by a senior member of the research team. They were attended by 5 to 17 participants and were approximately one hour in length.

Semi-structured telephone interviews were conducted with staff based outside of Melbourne. These were conducted at a time convenient to the participants by a member of the research team. Telephone interviews were also approximately one hour in length. 
A schedule of questions was used (Table 1) for both focus groups and interviews, with the ability to explore emerging topics as these arose. All interviews and focus groups were audio-recorded and transcribed verbatim and the interviews were continued until saturation of themes was reached.

\section{Data Analysis}

A qualitative thematic analysis [12] was undertaken by three researchers independently and compared for inter-rater reliability. Agreement between the initial reviewers was reached for all components of analysis. The emergent themes were then discussed by the wider research team and consensus was reached on themes and sub-themes. When a repetition of themes occurred and no new themes were found, the cohort was considered complete.

Here we report the emergent themes related to interdisciplinary interactions that were made explicit in the care of patients with advanced cancer in the ED. Environmental issues influencing care are reported elsewhere [13].

Approval was obtained from the institutional human research ethics committee prior to study commencement.

\section{Results}

\section{Participants}

Six focus groups were conducted in two quaternary referral metropolitan hospitals. Two focus groups were conducted with community palliative care clinicians from two metropolitan community palliative care services, each of which cover a large area of Melbourne, Australia extending from the inner city to the outer suburbs. In total there were 83 focus group participants, whose occupational roles and work locations are shown in Table 2. These focus groups were supplemented with 11 semi-structured interviews with emergency doctors and nurses from Australian States and Territories outside Victoria, whose occupations are shown in Table 2.

\section{'Ideal care' versus the realities of practice}

The over-arching theme to emerge was one of a conflict between a view of 'ideal care' and the realities of practice, particularly arising where clinicians from different services were required to work together to provide care (Table 3). For example, although all interviewees reported the importance of high quality communication of information between services, the realities of practice meant that this was often compromised by other service-based imperatives. The sub-themes comprised communication, decision-making, and the understanding of other services.

\section{Communication}

Participants from all services reported that high quality communication between services and departments was required for optimal care. Staff expected medical information about the illness and 
treatment, but also broader psychosocial information, such as the patient's understanding of the illness to be communicated.

I'm always looking for a letter from the oncologist, the treating oncologist so you know what to do. Sometimes they write it [in] their correspondence; ...whatever they have discussed with the patient or what their feelings were regarding the treatment. (ED doctor)

However, in practice, despite all participants reporting the need for high quality communication, gaps in information transfer were identified and appeared to affect most interactions. Emergency clinicians reported receiving little information from oncologists or palliative care services when patients were referred. Meanwhile, PC clinicians and oncologists reported an, at times, hostile response from ED staff when telephoning through information, which resulted in some not calling at all.

... I don't know what the doctor thinks they [the patient] should be understanding because I don't have access to their notes and letters. (ED clinician)

I often find... I get a response from the [ED] triage nurse that is, "well why are you telling me all of this, they're just going to be treated in order anyway so don't, don't bother". (PC clinician)

Community PC staff reported not receiving information after sending patients to the ED for assessment. However, at the same time they reported difficulty transmitting written information to ED outside usual business hours (9am to $5 \mathrm{pm}$ on weekdays) and were hesitant about providing telephone contact details after hours for fear of being overwhelmed with calls.

...that is often dangerous giving out the [after hours on call] pager number..... Yes, sometimes you give that very reluctantly. (PC clinician)

Oncologists reported they did not wish to provide extensive written information about their patients, preferring to be contacted by ED and thereby guide treatment decisions. However, ED doctors reported difficulty contacting the 'right' oncologist after hours. Whilst the ED doctors reported seeking guidance regarding management and goals of care from oncologist's clinic letters in the medical records, the oncologists viewed these clinic letters as a record for their own service and instead preferred to be directly contacted for management decisions to minimise ambiguity or misinterpretation of treatment intent.

I don't think anyone could make a prognostic judgement from reading that letter [oncology clinic letter]. (Oncologist)

\section{Decision-Making}

Participants from all services expressed the view that discussions about goals of care and important management decisions should ideally occur with a doctor who knew the person with advanced cancer 
well, usually an oncologist or palliative care physician. Advance care plans for people with advanced cancer, with established goals of care and plans made for potential complications, were advocated.

Well I think that's [goals of care] a decision for the oncologist with the patient, it's certainly not an ED decision. (ED clinician)

However, in reality ED physicians were often required to make decisions about treatment and treatment escalation when advanced cancer patients presented to the ED.

...we sort of had an idea that she was probably progressive and probably wasn't really appropriate for quite aggressive management. But, that decision wasn't really clear and it wasn't really made, so we were left in no man's land. (ED clinician)

As previously reported [13], ED staff commented that many patients seemed to lack awareness of their prognosis and treatment intent, suggesting advance care planning discussions and decisions were not being undertaken. They were critical of cancer specialists for not seeming to have explicit discussions and plans in place.

A lot of the times I've seen patients [that] have a palliative diagnosis [and] nobody's had that discussion ... and that becomes a really difficult situation at times to then bring it up after seeing them for ten minutes. (ED clinician)

Oncologists pointed to the complexities and challenges of discussing prognosis and goals of care. They described this planning as a process occurring over a period of time and often not appropriate when first meeting a patient. Furthermore the variety and complexity of problems associated with advanced cancer meant it was not possible to plan for all eventualities.

I can't predict every single thing that might go wrong in the patient, and so I might say, "Oh if SVC obstruction we'll do this. If you start getting haemorrhagic crisis we'll do this" but I might not have covered the PE, I might not have covered the big heart attack, so I might not have covered every single thing, so in ED they're quite like "oh your patient has got a massive PE. You didn't specify that in your letter" (Oncologist)

Some participants suggested that, in contrast to the agreed idea of best care, longstanding relationships between physicians and their patients may actually make decisions about treatment limitations more difficult. They noted that ED doctors, as independent physicians, were perhaps better placed to provide an objective perspective in some situations.

You almost do wonder, whether ... each patient, with a potentially incurable disease actually needs an independent doctor, who is not involved with them, to actually discuss end of life issues with them. (ED clinician) 


\section{Understanding of Other Services}

Participants from all services reported that optimal care for advanced cancer patients would involve remaining in the community and avoiding ED attendance and hospital admission. ED attendance was widely seen as a failure and the ED a less than ideal environment for patients with advanced cancer.

I think we go to great lengths to avoid presentations to emergency departments unless we really have to. (PC clinician)

Various services working together as a team was also widely considered a requirement for high quality care. However, participants were often critical of aspects of care provided in other areas and appeared to lack understanding of the demands placed on other services, and the role and skills of healthcare professionals working in other areas.

PC clinicians viewed their patients attending the ED as a negative outcome and were at times critical of ED waiting times and pain management. Despite this they relied upon the ED to provide initial assessment and management of patients known to their services after-hours, in acute crises, and when no inpatient palliative care beds were available. They also recalled patients who had been well managed by ED staff.

I also had one lady that was terminal, the family weren't coping, wasn't able to get direct admit into a palliative care unit, and was actively dying on admission to the emergency department. And the emergency department really did a great job. (PC clinician)

The PC services perceived a lack of understanding of their service, particularly what they were able to provide in the community. This was demonstrated in one focus group where the ED nurses were not aware that a 24-hour on call PC service was available in their hospital.

a lot of people .... they don't know where the heck we're from. (PC clinician)

Oncologists, whilst acknowledging urgent decisions might need to be made in ED on occasions, were uncomfortable with the concept of an emergency physician making decisions about treatment approaches for their patients. They were not confident that directives and discussions of goals in the medical record would be understood or enacted appropriately and therefore they wished to be actively involved with treatment decisions for their patients.

I don't think it's appropriate for them to be having discussions on limitations of care..., I would feel uncomfortable if they're having those kind of discussions to be fair, .... You know if someone's clearly deteriorating in front of your eyes and you need to make a call it's a whole different ball game, then it's fair and I agree that's their role. (Oncologist) 
However, while the oncologists were uncomfortable with ED physicians making management decisions, they did expect the ED to provide initial assessment of known oncology patients when they presented the ED.

...if we can avoid it we get the patient to come straight to clinic. .... [if] for some reason that's not feasible, we let them do the second sort of fall-back position [which] is coming to ED, so you would then you expect that ED is going to do the initial assessment. (Oncologist)

The oncologists' thought that ED clinicians may not have much experience caring for dying patients. However, as we have previously reported, ED clinicians in this study felt comfortable caring for dying patients and reported finding this rewarding [13]. They recognised the importance of timely pain relief and strived to facilitate privacy and space for family if a person was dying.

If someone is dying and we can't get them upstairs we really do try and put them [in the single room] where their family can sit with them. (ED clinician)

\section{Discussion}

Our study demonstrates commonality of views across health care disciplines of the constituents of optimal care in the setting of advanced cancer; and that this involved (1) high quality communication, (2) planning care and decision making by those health care providers most involved in the patient's care, and (3) community based care with ED to be avoided if possible. However, there was frequently discord between these ideals and the actual care provided. Service demands and the day-to-day stressors of practice appeared to influence people's actions and, perhaps unknowingly, engendered conflict with these ideals. For example, while good communication between services was advocated, barriers to this were imposed by staff. This resulted in tensions arising when services were required to work together to provide care, with participants at times critical of other services for not practising this ideal yet not recognising how they themselves may also be hindering this.

Previous research in this area has identified environmental [10], work-load [10], and cultural barriers [11] to the provision of end-of-life care in ED. Interdisciplinary interactions however, have been little considered in the care of people with advanced cancer in the ED. In other areas of cancer care, research has identified poor communication between GPs and oncologists [7] and whilst multidisciplinary cancer meetings have been demonstrated to improve collegial communication and interaction [14, 15, 16], barriers persist. Allied health and nursing team members are less likely to contribute to meeting discussions and report perceiving their input is not respected [14]. More broadly, barriers to interdisciplinary teamwork have been indentified in a range of healthcare settings including operating theatres [17] and intensive care [18], with limited understanding of roles and capabilities across interdisciplinary boundaries identified [19].

Within hospitals, doctors and other health professionals often work confined to their own specialty areas with little interaction or communication with other areas. This results in challenges when 
patients, such as those with advanced cancer, require care across a number of different services. With most relationships and interactions occurring within the discipline (whether it is surgery, oncology or other), there is less interaction and therefore less well developed relationships and consequent trust with those from other disciplines. This may translate to limited understanding of what such professionals may contribute. Whilst the lack of recognition of general practitioners' role in care has been reported [20], in our study a lack of recognition by other health care professionals of the skills ED physicians have in decision-making and caring for the dying was identified.

Complex patients, such as those with advanced cancer require substantial care coordination as they negotiate different complications of their illness. Many advanced cancer patients attend the ED during the course of their illness and treatment. Emergency clinicians should be viewed as part of this team. As such their input should also be sought when cancer services are planned and developed, and their role considered when engaging in advance care planning with patients.

Research on interdisciplinary interactions has frequently focused on practical ways of improving communication, such as electronic access to medical records and electronic discharge summaries automatically transmitted to general practitioners on completion, which have been shown to aid communication [21, 22]. However, this study has highlighted factors underlying communication gaps, namely a lack of understanding of the demands and stressors placed on colleagues in other areas and a lack of appreciation of their role and skills. If improvements in communication are to occur, this underlying lack of understanding needs to be addressed. Whilst medical letters may become more easily accessed through on-line records, unless there is concurrent improved trust of collegial judgement, there is unlikely to be any change in the information content and therefore the utility of those letters.

Improved understanding and trust could be enhanced by combined education sessions, such as case discussions following the transition through the ED of a person with advanced cancer and complex care needs. This current study has proceeded as an interdepartmental research project and has had an unanticipated outcome of improved interaction and understanding between the involved departments in our institution and, in turn, led to further interdepartmental educational and research activities. Communication skills are taught to healthcare students and professionals and emphasised as central to providing high quality clinical care to patients. Teaching of these skills could be broadened to encompass communication with healthcare colleagues. Finally, promoting a culture of collegial respect, through senior leadership and modelling is likely to improve interdisciplinary interactions hospital wide.

It is likely that the widely held notions of 'ideal care' may not be universally achievable in the real world. For example, discussions with people with advanced cancer about their wishes and goals of care, and planning for potential problems may certainly be beneficial, but may not always be possible. Uncertainty around disease stage and prognosis often exist, events may arise before the opportunity to discuss them has occurred, patients, families and even their doctors, may not be ready to embark on confronting discussions, and the complexities of advanced cancer make planning for every 
eventuality impossible. The idea that all advanced cancer patients should be able to be managed in the community may also be unachievable, as even with the best planning, unexpected or difficult to manage symptoms may arise, or at a time of crisis family members may be overwhelmed and prefer to go the ED. Expecting these ideals to be universally achievable reflects a failure to fully recognise the broad psychological, social and physical complexities faced by people with advanced cancer.

Our study had several limitations. In a focus group some participants may feel reluctant to express views which differ from those of the broader group, however, additional individual interviews were also included. Experiences of healthcare professionals working in Australia may differ from those working in other countries due to differences in healthcare service delivery, training and culture. The study was exploratory in nature with the qualitative method and sampling seeking to reveal a wide spectrum of views and ideas. This method precludes generalizability of results to a broader group of health care professionals. A quantitative method would be required to determine the extent to which such views are held.

The concept of 'ideal care' and agreement across specialities of the nature of what is ideal, suggests a useful concept in developing health care services and improving practice. However the gaps between what is considered ideal and usual practice existed and appeared to be embedded in a system which limits the understanding of the nature, practice and stressors that occur in other disciplines. All healthcare professionals interviewed showed great care and understanding for their patients with advanced cancer, and attempted to do their best to provide optimal care; thus all ultimately had similar goals. Improving care further is likely to involve improving our interdisciplinary relationships.

\section{Acknowledgements:}

This study was supported by the Victorian Cancer Agency. This study was also supported by the Australasian College for Emergency Medicine and College of Emergency Nursing Australia, the views of these researchers do not necessarily represent the views of these Colleges.

\section{Conflict of interest}

The authors declare that they have no conflict of interest relevant to this manuscript. The authors have control of all primary data and agree the journal may review this if requested. 
References:

1. Department of Health (2000) The NHS Cancer Plan: A Plan for Investiment, a Plan for Reform. Department of Health, London.

2. National Breast and Ovarian Cancer Centre (2010) Multidisciplinary Meetings for Cancer Care: A Guide for Healthcare Providers. Australian Government Department of Health and Ageing, Canberra.

3. ASCO-ESMO (2006) ASCO-ESMO consensus statement on quality cancer care. J Clin Oncol 24: 3498-3499

4. Spiegel W, Zidek T, Karlic H, Maier M, Vutuc C, Isak K, Micksche M (2010) Cancer patients' perception of information exchange between hospital-based doctors and their general practitioners. J Eval Clin Pract 16: 1309-1313

5. Karapinar F, van den Bemt PM, Zoer J, Nijpels G, Borgsteede SD (2010) Informational needs of general practitioners regarding discharge medication: content, timing and pharmacotherapeutic advice. Pharm World Sci 32: 172-178

6. Garasen H, Johnsen R (2007) The quality of communication about older patients between hospital physicians and general practitioners: a panel study assessment. BMC Health Serv Res 7: 133

7. McConnell D, Butow PN, Tattersall MH (1999) Improving the letters we write: an exploration of doctor-doctor communication in cancer care. Br J Cancer 80: 427-437

8. McKenzie H, Hayes L, White K, Cox K, Fethney J, Boughton M, Dunn J (2011) Chemotherapy outpatients' unplanned presentations to hospital: a retrospective study. Support Care Cancer 19: 963-969

9. Philip J, Collins A, Brand C, Gold M, Lethborg C, Murphy M, Moore G, Bohensky M Sundararajan V (2012) Utilising hospital data sets to identify patterns of service use in patients with a primary malignant glioma. Abstract Listing. Asia Pac J Clin Oncol 8: 189

10. Beckstrand RL, Smith MD, Heaston S, Bond AE (2008) Emergency nurses' perceptions of size, frequency, and magnitude of obstacles and supportive behaviors in end-of-life care. $\mathrm{J}$ Emerg Nurs 34: 290-300

11. Grudzen CR, Richardson LD, Hopper SS, Ortiz JM, Whang C, Morrison RS (2012) Does palliative care have a future in the emergency department? Discussions with attending emergency physicians. J Pain Symptom Manage 43: 1-9

12. Grbich C (2007) Qualitative data analysis : an introduction. SAGE Publications, London ; Thousand Oaks, Calif.

13. Jelinek GA, Marck CH, Weiland TJ, Philip J, Boughey M, Weil J, Lane H (2013) Caught in the middle: Tensions around the emergency department care of people with advanced cancer. Emerg Med Australas 25: 154-160

14. Devitt B, Philip J, McLachlan SA (2010) Team dynamics, decision making, and attitudes toward multidisciplinary cancer meetings: health professionals' perspectives $\mathrm{J}$ Oncol Pract 6: e17-20

15. Gagliardi AR, Wright FC, Anderson MA, Davis D (2007) The role of collegial interaction in continuing professional development J Contin Educ Health Prof 27: 214-219

16. Harrison JD, Choy ET, Spillane A, Butow P, Young JM, Evans A (2008) Australian breast cancer specialists' involvement in multidisciplinary treatment planning meetings Breast 17: 335-340

17. Lingard L, Reznick R, Espin S, Regehr G, DeVito I (2002) Team communications in the operating room: talk patterns, sites of tension, and implications for novices Academic medicine : journal of the Association of American Medical Colleges 77: 232-237

18. Thomas EJ, Sexton JB, Helmreich RL (2003) Discrepant attitudes about teamwork among critical care nurses and physicians Critical care medicine 31: 956-959

19. Weller JM, Janssen AL, Merry AF, Robinson B (2008) Interdisciplinary team interactions: a qualitative study of perceptions of team function in simulated anaesthesia crises Medical education 42: 382-388

20 Evans K, Mayer J, Treloar C (2000) Who cares? A focus group study on follow up care after discharge from hospital. Aust Fam Physician 29: 384-388

21 Alderton M, Callen J (2007) Are general practitioners satisfied with electronic discharge summaries? HIM J 36: 7-12

22 O'Leary KJ, Liebovitz DM, Feinglass J, Liss DT, Evans DB, Kulkarni N, Landler MP, Baker DW (2009) Creating a better discharge summary: improvement in quality and timeliness using an electronic discharge summary. J Hosp Med 4: 219-225 
Table 1 Focus group discussion guide

\section{ED care experiences}

1 Tell me about the last patient with advanced cancer you cared for in / directed to the ED.

\section{Perceptions of advanced cancer patients}

2. If you become aware that a patient is known to a community palliative care service what does that mean? What are the implications?

\section{Role of ED in the care of patients with advanced cancer}

3. What do you see as the role of the ED in the care of patients with advanced cancer?

4. Do you feel ED professionals are adequately prepared and supported in caring for patients with advanced cancer?

\section{Communication}

5. Can you tell me about a case where communication went well? And a case where it didn't go as well as you may have hoped or anticipated?

\section{Challenges}

6. Are there any challenges in caring for / directing patients advanced cancer in the ED?

\section{Rewards}

7. What are the positive outcomes of caring for patients with advanced cancer in the ED?

\section{Targeting future interventions}

8. If you think about the future, and the aim of improving care in the ED for patients with advanced cancer, what do you see as the priorities for intervention? 
Table 2 Discipline and work location of study participants

\begin{tabular}{lll}
\hline Focus Group Participants $(\mathrm{n}=83)$ & & 22 \\
\hline Hospital ED & Nurse & 21 \\
& Doctor & 25 \\
Community Palliative Care & Nurse/ Allied Health & 3 \\
& Doctor & 5 \\
Palliative Care & Nurse/ Allied Health & 1 \\
Hospital Oncology & Doctor & 0 \\
& Nurse & 6 \\
\hline Interview Participants $(\mathrm{n}=11)$ & Doctor & \\
\hline & & 3 \\
\hline
\end{tabular}


Table 3 Summary of subthemes

\begin{tabular}{lll}
\hline $\begin{array}{l}\text { Overarching } \\
\text { Theme }\end{array}$ & Subthemes & Ideal care \\
\hline $\begin{array}{l}\text { 'Ideal care' } \\
\text { versus the } \\
\text { realities of } \\
\text { practice }\end{array}$ & Communication & $\begin{array}{l}\text { High quality communication between } \\
\text { healthcare providers involved in a } \\
\text { person's care is regarded as } \\
\text { necessary for provision of best care. }\end{array}$ \\
& \\
& Decision Making \\
& $\begin{array}{l}\text { A physician who knows the patient } \\
\text { well is best placed to have } \\
\text { discussions about future care. } \\
\text { All advanced cancer patients should } \\
\text { have had discussions about goals of } \\
\text { care and advance care planning. }\end{array}$ \\
&
\end{tabular}

Realities of practice

Communication was hindered by practices of health care professionals.

ED clinicians report inadequate information received from oncology and $P C$, yet Oncology and PC clinicians report at times receiving a hostile response when telephoning ED with information.

Oncologists did not see clinic letters as providing guidance for ED and preferred verbal communication.

Referring services (PC and Oncology) believed they should be involved in patient management decisions, but were difficult to contact.

Oncologists were sometimes unresponsive to ED requests for admission.

ED clinicians often need to make urgent decisions about management approach.

ED clinicians critical about lack of advance care planning and a perceived lack of patient awareness of prognosis.

Oncologists report advance planning is a process and needs to be timed appropriately and that planning for every eventuality is not possible.

Decision making may be easier for an independent physician. 
Understanding of

Other Services
Advanced cancer patients should be able to be cared for in the community. ED attendance suggests service failure.

We all work together as a team to provide patient centred care.
PC clinicians critical of aspects of ED, but often relied on ED for initial assessment and management of patients.

Oncologists uncomfortable with ED physicians making decisions but also expected them to provide initial assessment.

There was a lack of recognition of the experience ED clinicians have in caring for dying patients.

There was a lack of understanding of availability and role of PC services. 